\title{
PRODUTO INTERNO BRUTO DO SETOR FLORESTAL BRASILEIRO, 1994 A 2008 $^{1}$
}

\author{
Naisy Silva Soares², Márcio Lopes da Silva³ e Sidney Araujo Cordeiro ${ }^{4}$
}

\begin{abstract}
RESUMO-Este trabalho analisou e estimou, conforme metodologia apresentada em Passos e Nogami (2006), o Produto Interno Bruto (PIB) do setor florestal brasileiro, no período de 1994 a 2008. Os resultados indicaram que o setor florestal contribuiu para o PIB brasileiro, mas essa contribuição diminuiu ao longo dos anos; o segmento da madeira processada mecanicamente foi o que mais contribuiu para o PIB do setor florestal; o aumento no PIB nominal foi causado, em grande parte, pelo incremento dos preços dos produtos florestais; e o segmento de produtos florestais não madeireiros perdeu participação no PIB do setor florestal.
\end{abstract}

Palavras-chave: Agregado econômico; Crescimento econômico; Economia florestal.

\section{GROSS DOMESTIC PRODUCT OF THE BRAZILIAN FOREST SECTION, 1994 TO 2008}

\begin{abstract}
The present work analyzed and calculated, according to the methodology presented in Passos and Nogami (2006), the gross domestic product (GDP) of the Brazilian forest section, from 1994 to 2008. The results indicated that the forest section contributed to the brazilian GDP, but that contribution has reduced along the years; the segment of mechanically processed wood was the one that contributed the most to the GDP of the forest section; the increase in nominal GDP was caused, most of the times, by the increase of the prices of the forest products; and the segment of non-wood forest products lost participation in GPD of the forest section.
\end{abstract}

Keywords: Economic aggregate; Economical growth; Forest economy.

\section{INTRODUÇÃO}

Entende-se por setor florestal todo o conjunto de atividades produtivas primárias e secundárias que exploram, conservam, manejam e renovam e, ou, implantam florestas, bem como utilizam a madeira como principal insumo na transformação industrial (BREPOHL, 1980).

Esse setor começou se destacar no país a partir de 1965, quando foram criados os incentivos fiscais ao reflorestamento, o que possibilitou às empresas abaterem até 50\% do valor do imposto de renda devido para ser aplicado em projetos florestais. Em decorrência da legislação (Lei n 5.106, de setembro de 1966), o crescimento da área reflorestada no país situou na faixa de 100 a 250 mil hectares anuais entre 1968 e 1973, elevando-se para 450 mil hectares anuais de 1974 a 1982. Em 1976, o Brasil era um dos quatro países que mais incentivavam a produção florestal, depois da China, União Soviética e Estados Unidos. Ressalta-se, ainda, que os projetos vinculados à política de incentivos fiscais totalizaram, aproximadamente, 6,2 milhões de hectares entre 1967 e 1986 (LEÃO, 2000).

Em 1988, foram extintos os incentivos fiscais ao reflorestamento devido às deficiências técnicas na instalação e distorções na aplicação de recursos disponíveis. A produção de muitos projetos florestais

\footnotetext{
${ }^{1}$ Recebido em 23.09.2010 aceito para publicação em 05.06.2014

${ }^{2}$ Departamento de Ciências Econômicas da Universidade Estadual de Santa Cruz, UESC, Brasil. E-mail: < naisysilva@yahoo.com.br> .

${ }^{3}$ Departamento de Engenharia Florestal da Universidade Federal de Viçosa, UFV, Brasil. E-mail: <marlosil@ufv.b>.

${ }^{4}$ Departamento de Engenharia Florestal da Universidade Federal dos Vales do Jequitinhonha e Mucuri, UFVJM, Brasil. Email:<sidneyufv@yahoo.com.br>.
} 
implantados ficou abaixo das expectativas, em termos de qualidade e quantidade. Mas, mesmo com o fim dos incentivos fiscais, esse setor continuou se desenvolvendo no país, porém com as grandes empresas de base florestal dedicando-se à ampliação de sua área reflorestada, com recursos próprios ou tomando empréstimos de longo prazo em bancos de fomento estaduais ou federais, como o Banco de Desenvolvimento do Paraná S/A (BADEP) e Banco Nacional de Desenvolvimento Econômico e Social (BNDES), ou incentivando o reflorestamento em pequenos e médios imóveis rurais (ANTONANGELO; BACHA, 1998; LEÃO, 2000).

Em 2008, a área com florestas nativas no Brasil foi da ordem de 540 milhões de hectares e a com povoamentos plantados, 6,5 milhões de hectares. Em termos de florestas plantadas, os gêneros que mais se destacaram foram o eucalipto e o pinus, representando $64,7 \%$ e $28,4 \%$ do total, respectivamente. O Estado com maior área plantada de eucalipto foi Minas Gerais, com $29 \%$ do total, seguido por São Paulo (22\%), Bahia (14\%) e Rio Grande do Sul (7\%). Os demais estados juntos responderam por 29\%. Com relação à área plantada de pinus, os Estados da Região Sul foram os que mais se destacaram. O Paraná respondeu por 38\% da área plantada com o gênero, Santa Catarina 30\% e o Rio Grande do Sul 9\%. Os outros Estados representaram $23 \%$ do total (ASSOCIAÇÃO BRASILEIRA DE PRODUTORES DE FLORESTAS PLANTADAS, 2009).

A relevância do setor florestal para a economia brasileira, em termos de geração de renda, emprego, impostos e divisas, foi comprovada em vários estudos científicos realizados a partir do ano 2000, como os de Valverde (2000), Martins et al. (2003), Silva (2004), Valverde et al. (2005a), Valverde et al. (2005b) e Sousa et al. (2010).

De modo geral, nesses estudos foi constatado que o setor florestal configurou-se como setor-chave na economia em determinado ano, pois teve considerável poder de compra; apresentou melhor desempenho em produção, emprego, renda e arrecadação de impostos do que importantes setores econômicos; e contribuiu para o saldo positivo da balança comercial brasileira. Contudo, até o momento, há poucos estudos analisando a participação do setor florestal no Produto Interno Bruto (PIB) do país, bem como o comportamento do PIB florestal brasileiro ao longo dos anos (BREPOHL, 1980; PAULA et al., 1996).

Revista Árvore, Viçosa-MG, v.38, n.4, p.725-732, 2014
Com isso, torna-se relevante atualizar pesquisas nessa área, para entender a contribuição desse setor na economia ao longo dos anos e auxiliar na formulação de políticas públicas que visem favorecer o crescimento do setor e, consequentemente, do país, uma vez que o PIB é um indicador de crescimento econômico. Além disso, há dificuldades em estimar o PIB florestal devido à indisponibilidade de dados, falta de conhecimento sobre o conceito e o método usado para cálculo do PIB real e PIB nominal e divergência dos dados das diferentes fontes.

Nesse contexto, este trabalho teve como principal objetivo analisar o PIB do setor florestal brasileiro, no período de 1994 a 2008.

Especificamente, pretendeu-se estimar o PIB do setor florestal no país e dos seus principais segmentos no período supracitado; verificar a contribuição do setor florestal para o PIB brasileiro e a contribuição dos seus segmentos ao PIB do setor; e propor políticas que podem contribuir para o crescimento do PIB do setor florestal nacional.

\section{MATERIAL E MÉTODO}

\subsection{Referencial analítico}

\subsubsection{Estimativa do PIB}

O PIB é um dos indicadores mais utilizados na economia para mensurar a atividade econômica de uma região ou país. Representa a soma, em valores monetários, de todos os bens e serviços finais produzidos em determinada região, sejam países, estados e cidades, durante determinado período de tempo, independentemente da nacionalidade dos proprietários das unidades produtoras desses bens e serviços (MANKIW, 1999; FROYEN, 2001).

Para analisar o PIB ao longo do tempo, é necessário distinguir entre PIB nominal e PIB real. O PIB nominal refere-se ao valor do PIB calculado a preços correntes (equação 1), e o PIB real está relacionado com o valor do PIB calculado a preços constantes (equação 2) (PASSOS; NOGAMI, 2006).

$$
P I B_{\text {no min } a l}=\sum_{i}^{n}\left(P_{i n} x Q_{\text {in }}\right)
$$

em que $\mathrm{P}=$ preço, $\mathrm{Q}=$ quantidade produzida, $\mathrm{i}$ $=$ produto e $\mathrm{n}=$ período. 


$$
P I B_{\text {real }}=\left(\frac{P N B_{\text {no min } a l}}{\text { Índice de } \operatorname{Pr} e c ̧ o}\right) x 100
$$

O mais indicado é o uso de seu valor a preços constantes, que leva em conta apenas as variações nos volumes produzidos dos bens e não as alterações de seus preços de mercado.

De acordo com Passos e Nogami (2006), ao medir o PIB de um país surge um grande problema, que é a possibilidade de dupla contagem, ou seja, de se computar mais de uma vez um bem no PIB, acabando por superestimá-lo. Assim, os bens intermediários devem ser excluídos do cálculo, pois eles já estão incluídos no valor do produto final.

Por exemplo: considera-se o valor do automóvel como parte do PIB. Mas excluem-se pneus, aço e vidro, pois esses itens já estão incluídos no preço do veículo. Entretanto, se os pneus, aço e vidro são exportados, eles são considerados bens finais e por isso devem entrar no cálculo do PIB. Assim, para calcular o PIB do setor florestal brasileiro, utilizaram-se as equações (1) e (2) e considerou-se o valor da produção nacional dos seguintes produtos: carvão vegetal, lenha, madeira serrada, aglomerado, compensado, MDF, chapas de fibra, lâminas, papel e papelão e produtos florestais não madeireiros.

No caso da celulose e da madeira em tora, considerou-se para cálculo o valor das exportações nacionais para não se incorrer em dupla contagem, haja vista que o preço da celulose está incluso no preço do papel e do papelão e o preço da madeira em tora, no preço dos produtos da madeira processada mecanicamente como madeira serrada, aglomerado, compensado, MDF, chapas de fibra e lâminas.

\subsubsection{Evolução do PIB Florestal Brasileiro}

A análise envolvendo a evolução do PIB foi realizada por meio da taxa geométrica de crescimento (TGC), calculada como demonstrado a seguir (GUJARATI, 2000).

$$
Y=a \cdot b^{T}
$$

em que $\mathrm{Y}=$ variável dependente, $\mathrm{T}=$ tempo e a e b = parâmetros a serem estimados .

Aplicando a forma logarítmica, tem-se (equação 4):

$$
\log Y=\log a+T \log b
$$

Desse modo, a TGC é obtida pela equação 5.

$$
T G C=(\text { Anti }-\log b-1) \times 100
$$

\subsection{Fonte de dados}

Os dados utilizados para cálculo do PIB do setor florestal brasileiro são provenientes de séries temporais anuais, abrangendo o período de 1994 a 2008. Não se trabalhou com um período maior porque alguns dados não estavam disponíveis e por entender que o período abrangido é representativo e capta a evolução do setor.

As séries foram construídas como indicado a seguir:

O valor da produção nacional do carvão vegetal, lenha e dos produtos florestais não madeireiros são do Instituto Brasileiro de Geografia e Estatística (IBGE), em que este último se refere ao valor da produção da extração vegetal coletados pelo Instituto (IBGE, 2010).

Já o valor da produção da madeira serrada, aglomerado, compensado, MDF, chapas de fibra, lâminas e papel e papelão foi obtido na Food And Agriculture Organization (FAO), assim como o valor das exportações de celulose e madeira em tora (FAO, 2010).

Ressalta-se que para calcular o valor da produção dos produtos florestais madeireiros no Brasil foi utilizado o preço de exportação como proxy do preço médio anual desses produtos no país, devido à indisponibilidade de dados, com exceção da celulose. Para esse produto, optou-se por utilizar os preços da celulose coletado pelo Centro de Estudos Avançados em Economia Aplicada - CEPEA (CEPEA, 2010).

Os dados sobre o PIB do Brasil, utilizados para verificar a contribuição do PIB florestal ao PIB brasileiro, são do IBGE e estão disponíveis no site do Instituto de Pesquisa Econômica Aplicada - IPEA (IPEA, 2010).

Para calcular o PIB real, foi utilizado o Índice Geral de Preços - Disponibilidade Interna (IGP-DI) (1994 = 100) da Fundação Getúlio Vargas (FGV), também disponível no site do IPEA (IPEA, 2010).

\section{RESULTADOS}

\subsection{Estimativas do PIB}

As estimativas do PIB do setor florestal e dos segmentos de produtos florestais não madeireiros, celulose e papel e madeira processada mecanicamente, de 1994 a 2008, podem ser verificadas na Tabela 1, assim

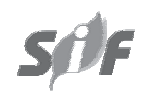

Revista Árvore, Viçosa-MG, v.38, n.4, p.725-732, 2014 
Tabela 1 - PIB do setor florestal brasileiro e de seus segmentos, 1994 a 2008, em milhões de R\$.

Table 1 - GDP of the brazilian forest section and its segments, 1994 to 2008, in millions of R\$.

\begin{tabular}{|c|c|c|c|c|c|c|c|c|c|c|c|c|}
\hline \multirow[t]{2}{*}{ Ano } & \multicolumn{3}{|c|}{ Setor Florestal* } & \multicolumn{3}{|c|}{$\begin{array}{c}\text { Produtos Florestais } \\
\text { Não-Madeireiros }\end{array}$} & \multicolumn{3}{|c|}{ Celulose e Papel } & \multicolumn{3}{|c|}{$\begin{array}{c}\text { Madeira Processada } \\
\text { Mecanicamente }\end{array}$} \\
\hline & $\begin{array}{c}\text { PIB } \\
\text { nominal }\end{array}$ & $\begin{array}{l}\text { PIB } \\
\text { real }\end{array}$ & $\begin{array}{c}\% \text { PIB } \\
\text { florestal }\end{array}$ & $\begin{array}{c}\text { PIB } \\
\text { nominal }\end{array}$ & $\begin{array}{l}\text { PIB } \\
\text { real }\end{array}$ & $\begin{array}{c}\% \text { PIB } \\
\text { florestal }\end{array}$ & $\begin{array}{c}\text { PIB } \\
\text { nominal }\end{array}$ & $\begin{array}{l}\text { PIB } \\
\text { real }\end{array}$ & $\begin{array}{c}\% \text { PIB } \\
\text { florestal }\end{array}$ & $\begin{array}{c}\text { PIB } \\
\text { nominal }\end{array}$ & $\begin{array}{l}\text { PIB } \\
\text { real }\end{array}$ & $\begin{array}{c}\% \text { PIB } \\
\text { florestal }\end{array}$ \\
\hline 1994 & 8.543 & 7.906 & 2,4 & 3.047 & 2.820 & 35,7 & 547 & 506 & 6,4 & 4.555 & 4.216 & 53,3 \\
\hline 1995 & 10.414 & 8.379 & 1,5 & 3.317 & 2.669 & 31,8 & 979 & 787 & 9,4 & 5.621 & 4.523 & 54,0 \\
\hline 1996 & 9.453 & 6.964 & 1,1 & 2.674 & 1.970 & 28,3 & 1.013 & 746 & 10,7 & 5.230 & 3.853 & 55,3 \\
\hline 1997 & 10.463 & 7.196 & 1,1 & 1.795 & 1.235 & 17,2 & 1.106 & 761 & 10,6 & 6.885 & 4.735 & 65,8 \\
\hline 1998 & 10.467 & 7.069 & 1,1 & 1.786 & 1.206 & 17,1 & 1.221 & 825 & 11,7 & 6.803 & 4.594 & 65,0 \\
\hline 1999 & 15.760 & 8.877 & 1,5 & 1.824 & 1.027 & 11,6 & 2.258 & 1.271 & 14,3 & 11.002 & 6.197 & 69,8 \\
\hline 2000 & 16.457 & 8.464 & 1,4 & 1.804 & 928 & 11 & 2.937 & 1.510 & 17,8 & 10.885 & 5.598 & 66,1 \\
\hline 2001 & 19.648 & 9.167 & 1,5 & 1.986 & 927 & 10,1 & 2.936 & 1.370 & 14,9 & 14.020 & 6.541 & 71,4 \\
\hline 2002 & 24.710 & 9.031 & 1,7 & 2.921 & 1.067 & 11,8 & 3.741 & 1.367 & 15,1 & 16.863 & 6.163 & 68,2 \\
\hline 2003 & 27.374 & 9.355 & 1,6 & 3.029 & 1.035 & 11,1 & 4.349 & 1.486 & 15,9 & 18.407 & 6.290 & 67,2 \\
\hline 2004 & 32.153 & 9.822 & 1,7 & 3.274 & 1.000 & 10,2 & 6.705 & 2.048 & 20,9 & 20.563 & 6.281 & 64,0 \\
\hline 2005 & 31.545 & 9.501 & 1,5 & 3.602 & 1.085 & 11,4 & 7.319 & 2.204 & 23,2 & 18.938 & 5.704 & 60,0 \\
\hline 2006 & 32.405 & 9.417 & 1,4 & 3.814 & 1.108 & 11,8 & 8.697 & 2.527 & 26,8 & 18.016 & 5.236 & 55,6 \\
\hline 2007 & 28.661 & 7.698 & 1,1 & 3.859 & 1.036 & 13,5 & 5.436 & 1.460 & 19,0 & 16.651 & 4.472 & 58,1 \\
\hline 2008 & 33.718 & 8.342 & 1,1 & 3.967 & 981 & 11,8 & 7.038 & 1.741 & 20,9 & 19.415 & 4.803 & 57,6 \\
\hline
\end{tabular}

Fonte: Dados da pesquisa. *Além dos três segmentos, incluem-se carvão vegetal e lenha.

Source: Research data. *In addition to the three segments, include vegetable charcoal and wood.

como a contribuição do setor florestal brasileiro ao PIB nacional e a contribuição dos segmentos de produtos florestais não madeireiros, celulose e papel e madeira processada mecanicamente ao PIB do setor florestal.

Como descrito anteriormente, a mais indicada é a análise do PIB real para identificar seu comportamento ao longo do tempo. Contudo, por motivo de comparação, o PIB nominal foi incluído neste trabalho.

\subsection{Evolução do setor florestal brasileiro}

Na Tabela 2, apresenta-se a TGC do PIB nominal e do PIB real do Brasil, do setor florestal e dos segmentos

Tabela 2 - Taxa Geométrica de Crescimento (TGC) do PIB, de 1994 a 2008.

Table 2 - Geometric Growth Rates (GGD) of the GDP, from 1994 to 2008.

\begin{tabular}{lcc}
\hline Variáveis & \multicolumn{2}{c}{ TGC (\% ao ano) } \\
\cline { 2 - 3 } & PIB nominal & PIB real \\
\hline Brasil & $12,3 *$ & $2,5 *$ \\
Setor Florestal & $11,1 *$ & $1,3 * *$ \\
Produtos Florestais & $3,7 *$ & $-6,0 *$ \\
não-madeireiros & $18,9 *$ & $9,1 *$ \\
Celulose e Papel & $11,3 *$ & $1,4 * *$ \\
Madeira Processada & & \\
Mecanicamente & & \\
\hline
\end{tabular}

Fonte: Dados da pesquisa. * Significativo a $1 \% ; * *$ significativo a $5 \%$; e*** significativo a $14 \%$.

Source: Research data. *Significant at 1\%; **significant at 5\%; and $* * *$ significant at $14 \%$. que o compõem, que caracterizam a evolução do setor no período de 1994 a 2008.

\section{DISCUSSÃO}

\subsection{Estimativas do PIB}

Com base nos resultados apresentados na Tabela 1 , nota-se que o PIB nominal do setor florestal e dos seus segmentos aumentou no período sob análise. Mas, quando é considerado o PIB real, verifica-se que o crescimento do PIB do setor florestal e dos segmentos de celulose e papel e madeira processada mecanicamente foi menor em relação ao crescimento do PIB nominal. Além disso, verifica-se que houve redução no PIB do segmento de produtos florestais não madeireiros.

Isso indica que de 1994 a 2008 o aumento do PNB nominal foi causado, em grande parte, pelo aumento dos preços. Além disso, a preços constantes de 1994, o PIB do setor florestal aumentou de R \$7.906 milhões para R\$8.379 milhões, de 1994 para 1995, aumento esse de 6\%. Raciocínio análogo pode ser feito nos demais períodos e nos segmentos do setor florestal (Tabela 1).

A Tabela 1 mostra, ainda, que o segmento da madeira processada mecanicamente é o que mais contribuiu para o PIB do setor florestal brasileiro, seguido pelo segmento de celulose e papel e produtos florestais 
não madeireiros. Este último perdeu participação no PIB do setor florestal ao longo dos anos, ao contrário dos segmentos de celulose e papel e madeira processada mecanicamente.

A perda de participação do segmento de produtos florestais não madeireiros no PIB pode ser explicada pela falta de políticas governamentais favoráveis ao desenvolvimento da produção. A taxação elevada, a falta de linhas de créditos específicas para a atividade com taxas de juros compatíveis, a necessidade de política de preços e de taxa de câmbio favorável à atividade desestimulam o investimento da produção (ROSADO et al., 2006; SOUSA et al., 2009a,b).

No caso do segmento da madeira processada mecanicamente, o fato de esse segmento concentrar a maior parte das indústrias florestais explica a sua maior contribuição para o PIB do setor florestal. Como dito anteriormente, esse segmento engloba as indústrias da madeira serrada, aglomerado, compensado, MDF, chapas de fibra e lâminas.

Constatou-se, também com base na Tabela 1, que a contribuição do setor florestal ao PIB brasileiro diminuiu ao longo dos anos, o que pode estar relacionado com o maior crescimento do PIB brasileiro em relação ao PIB do setor florestal e ao fato de o PIB de outros setores ter contribuído mais para a economia nacional no período analisado que o PIB do setor florestal, conforme observado em Instituto de Pesquisa Econômica Aplicada (IPEA, 2010).

\subsection{Evolução do setor florestal brasileiro}

Verifica-se na Tabela 2 que o PIB nominal do setor florestal aumentou a uma taxa média anual de 11,1\%, e o PIB nominal dos segmentos de produtos florestais não madeireiros, celulose e papel e madeira processada mecanicamente também aumentou, em média, 3,7\%, $18,9 \%$ e $11,3 \%$ ao ano, respectivamente. Apenas o PIB nominal do segmento de celulose e papel apresentou TGC superior ao PIB nominal do Brasil.

No que diz respeito ao PIB real, pode-se dizer que o PIB real do Brasil cresceu o dobro do PIB real do setor florestal. Este apresentou crescimento médio anual de 1,3\%, enquanto o PIB real do Brasil cresceu, em média, 2,5\% ao ano (Tabela 2).

Considerando os segmentos do setor florestal, constatou-se que o PIB real do segmento de celulose e papel e da madeira processada mecanicamente cresceu
9,1\% e 1,4\%, respectivamente. No segmento de produtos florestais não madeireiros, ocorreu redução no PIB da ordem de 6\% ao ano, em média (Tabela 2).

A Tabela 2 mostra, também, que somente o segmento de celulose e papel apresentou taxa de crescimento do PIB real superior à do PIB real nacional.

Além do aumento dos preços da celulose e do papel, fatores que aumentaram a produção desses produtos no Brasil podem ter contribuído para o crescimento do PIB do segmento no período sob análise. Entre esses, cita-se a elevada produtividade das florestas de eucalipto no Brasil, que possibilita ciclos de rápido crescimento e alta qualidade; baixo custo de produção da celulose no Brasil em relação a outros países; e avanço tecnológico na área de genética, biotecnologia e manejo florestal.

No tocante à produtividade das florestas nacionais, pode-se dizer que, em 2007, a produtividade média das florestas nacionais de eucalipto alcançou $41 \mathrm{~m}^{3} / \mathrm{ha} /$ ano, enquanto na África do Sul, Chile, Portugal, Espanha, Uruguai e Indonésia essa produtividade corresponde a 20, 25, 12, 10, 25 e 20 m³/ha/ano, respectivamente. Acrescenta-se, ainda, que no Brasil a rotação das florestas de eucalipto é de sete anos, inferior à de países como África do Sul (8 - 10 anos), Chile (10 -12 anos), Portugal (12 - 15 anos) e Espanha (12 - 15 anos) (BRACELPA, 2009).

Com relação ao pinus, no Brasil a produtividade média das florestas de Pinus spp é de $35 \mathrm{~m}^{3} / \mathrm{ha}$ /ano. Já as florestas de Pinus radiata no Chile e na Nova Zelândia conseguem produzir $22 \mathrm{~m}^{3}$ /ha/ano de madeira. Nos Estados Unidos, a produtividade média das florestas de Pinus elliotti e de Pinus taeda é de $10 \mathrm{~m}^{3} / \mathrm{ha} / \mathrm{ano}$, e no Canadá a produtividade média das florestas de Pinus oregon é de $7 \mathrm{~m}^{3}$ /ha/ano. Entretanto, a rotação das florestas de pinus no Brasil é de 15 anos, enquanto no Chile, Nova Zelândia e Estados Unidos é de 25 anos e no Canadá, 45 anos (BRACELPA, 2009).

Observa-se, também, que enquanto no Brasil é necessária uma área de 100.000 ha para produção de um milhão de tonelada de celulose por ano, na Península Ibérica são necessários 300.000 ha e na Escandinávia, 720.000 ha (BRACELPA, 2009).

No que diz respeito aos custos totais de produção da celulose de fibra curta, os dados apresentados em Montebello (2006) corrigidos pelo IPA dos Estados

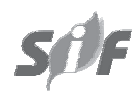

Revista Árvore, Viçosa-MG, v.38, n.4, p.725-732, 2014 
Unidos indicam que em 2009, no Brasil, esses custos foram cerca de US\$494/toneladas. Nos países de tradição florestal como Indonésia, Costa Leste do Canadá, Suécia, Finlândia, Portugal e Espanha, o custo total de produção de uma tonelada de celulose de fibra curta foi cerca de US\$ 439, US\$ 626, US\$ 604, US\$ 560, US\$ 549 e US\$ 604, respectivamente.

\subsection{Propostas para o crescimento do PIB do setor florestal brasileiro}

Acredita-se que fatores como a ineficiência das políticas públicas na tributação, legislação, taxa de câmbio, taxa de juros, nos encargos sociais e nas políticas comerciais podem explicar a redução da contribuição do setor florestal para o PIB nacional.

Como o PIB é um indicador de crescimento econômico, um crescimento do PIB do setor florestal brasileiro contribuiria para o crescimento da economia no país. Assim, recomendam-se as seguintes medidas para a expansão do PIB florestal:

- Políticas governamentais de longo prazo para aumentar a produção de produtos florestais no país e a área plantada.

- Aperfeiçoamento das linhas de financiamentos para aquisição de máquinas e equipamentos florestais, como o FINAME, pois as máquinas e equipamentos florestais possuem preços elevados e são demandados em todo o processo produtivo, ou seja, da etapa de produção da matéria-prima (madeira) até o produto final.

- Incentivo ao fomento florestal privado e aumento do número de produtores atendidos pelo fomento florestal público.

- Redução da taxa de juros para os projetos florestais, principalmente para o pequeno produtor rural.

- Melhoria em logística e infraestrutura, principalmente quanto a transportes, custos portuários e de energia.

- Eliminação da incidência de tributos em cascata ao longo das cadeias produtivas.

- Apoio ao desenvolvimento tecnológico, para expandir a produção florestal e as indústrias do setor no país, no sentido de manter a competitividade industrial no longo prazo e fortalecer o posicionamento das empresas no setor.
- Política cambial favorável às exportações de produtos florestais.

- Política de preços para os produtos florestais não madeireiros.

\section{CONCLUSÃO}

Conclui-se que o setor florestal possui contribuição significativa para o PIB brasileiro, a qual, porém, vemse reduzindo ao longo dos anos.

O segmento da madeira processada mecanicamente foi o que mais contribuiu para o PIB do setor florestal no decorrer dos anos. A partir de 1999, o segmento de celulose e papel apresentou a segunda maior contribuição para o PIB do setor, seguido pelo segmento de produtos florestais não madeireiros. Contudo, o segmento de celulose e papel apresentou a maior taxa de crescimento do PIB do setor no período estudado, seguido pelo segmento de produtos florestais não madeireiros e de madeira processada mecanicamente.

Verificou-se, também, que o aumento no PIB nominal do setor florestal e dos seus segmentos foi causado, em grande parte, pelo aumento dos preços dos produtos florestais e não pelo aumento da produção. No entanto, o segmento de produtos florestais não madeireiros perdeu a participação no PIB do setor florestal, enquanto os demais segmentos ganharam.

Sugere-se para outros trabalhos de pesquisa uma comparação entre o PIB do setor florestal brasileiro e o PIB de outros setores da economia, no sentido de verificar a contribuição de cada setor para o PIB nacional.

\section{AGRADECIMENTOS}

Ao Conselho Nacional de Desenvolvimento Científico e Tecnológico (CNPq) e à Fundação de Amparo à Pesquisa do Estado de Minas Gerais, pelo financiamento; e à Universidade Federal de Viçosa, pelo fornecimento da estrutura e pessoal.

\section{REFERÊNCIAS}

ANTONÂNGELO, A.; BACHA, C. J. I. As fases da silvicultura no Brasil. Revista Brasileira de Economia, v.52, n.1, p.207-238, 1998.

ASSOCIAÇÃO BRASILEIRA DE CELULOSE E PAPEL - BRACELPA. Setor de Celulose e

Papel. 2009. Disponível em: $<\underline{\text { http:// }}$ www.bracelpa.org.br> . Acesso em: 09 jun. 2010. 
ASSOCIAÇÃO BRASILEIRA DE PRODUTORES DE FLORESTAS PLANTADAS - ABRAF. (2009) Anuário Estatístico da ABRAF 2009 ano base 2008. Disponível em: $<\underline{\text { http:// }}$ www.abraflor.org.br/>. Acesso em: 10 de jun. 2010.

BREPOHL, D. Contribuição do Setor Florestal à Economia Brasileira. Revista Floresta, v.11, n.1, p.53-57, 1980.

\section{CENTRODEESTUDOSAVANÇADOSEM} ECONOMIAAPLICADA-CEPEA.(2010). Informativo CEPEA - Setor Florestal. Vários números. Disponível em: $<$ http:// www.cepea.esalq.usp.br>. Acesso em: 11 de maio 2010.

FOOD AND AGRICULTURE ORGANIZATION FAO. (2010). Disponível em: <http//:www.fao.org>. Acesso em: 23 de abr. 2010.

FROYEN, R. T. Macroeconomia. São Paulo: Saraiva, 2001. p.129-135.

GUJARATI, D. N. Econometria básica. 3.ed. São Paulo: Makron Books, 2000. 846p.

\section{INSTITUTO BRASILEIRO DE GEOGRAFIA E} ESTATÍSTICA - IBGE. (2010). Disponível em: <http/ /:www.ibge.gov.br>. Acesso em: 02 de maio 2010.

INSTITUTO DE PESQUISA ECONÔMICA APLICADA - IPEA. (2010). Disponível em: <http// :www.ipeadata.gov.br>. Acesso em: 02 de jun. 2010.

LEÃO, R. M. A floresta e o homem. São Paulo: Instituto de Pesquisas e Estudos Florestais, 2000. 448 p.

MANKIW, N. G. Introdução à economia: princípios de micro e macroeconomia. Rio de Janeiro: Campus, 1999.

MARTINS, G. et al. Inserção do setor florestal na estrutura econômica do Paraná. Revista Paranaense de Desenvolvimento, n.104, p.5-21, jan./jun. 2003.

MONTEBello, A. E. S. Análise da Evolução da Indústria Brasileira de Celulose no período de 1980 a 2005. 2006. 115f. Dissertação (Mestrado em Ciências - Área de Concentração: Economia Aplicada) - Escola Superior de Agricultura Luiz de Queiroz. Piracicaba, 2006.
PASSOS, C. R. M.; NOGAMI, O. Princípios de economia. 5.ed. São Paulo: Thomson Learning, 2006. p.526-533.

PAULA, R. A.; SILVA, M. L.; REZENDE, J. L. P. Formação do PIB Florestal Brasileiro e suas Controvérsias. In: SIMPÓSIO INTERNACIONAL SOBRE ECOSSISTEMAS FLORESTAIS, 4., 1996, Belo Horizonte - MG. Anais... Belo Horizonte: 1996.

ROSADO, P. L.; TOSTO, S. G.; GOMES, M. F. M. Competitividade e expansão da produção de borracha natural brasileira, no contexto de liberalização dos mercados. In: ALVARENGA, A. P. et al. Seringueira: aspectos econômicos sociais e perspectivas para o seu fortalecimento. Viçosa, MG: 2006. p.103-128.

SILVA, L. M. S. Relações intersetoriais da economia acreana e sua inserção na economia brasileira: uma análise insumoproduto. 2004. 184f. Dissertação (Mestrado em Ciência - Área de Concentração: Economia Aplicada) - Escola Superior de Agricultura "Luiz de Queiroz”, Piracicaba, 2004.

SOUSA, E. P. et al. Competitividade da Produção de Palmito de Pupunha (Bactris Gasipaes Kunth.) no Brasil In: CONGRESSO DA SOCIEDADE BRASILEIRA DE ECONOMIA, ADMINISTRAÇÃO E SOCIOLOGIA RURAL, 47., 2009, Porto Alegre. Anais..., Porto Alegre: SOBER, 2009a. CD ROM.

SOUSA, E. P. et al. Competitividade da Produção de Palmito de Pupunha no Espírito Santo e em São Paulo. In: ENCONTRO NACIONAL DA ASSOCIAÇÃO BRASILEIRA DE ESTUDOS REGIONAIS E URBANOS, 7., 2009, São Paulo. Anais... São Paulo: ENABER, 2009b. CD ROM.

SOUSA, E. P. et al. Desempenho do setor florestal para a economia brasileira: uma abordagem da Matriz Insumo-Produto. Revista Árvore, 2010 (no prelo).

VALVERDE, S. R. A contribuição do setor florestal para o desenvolvimento sócioeconômico: uma aplicação de modelos de equilíbrio multissetoriais. 2000. 105f. Tese (Doutorado em Ciência Florestal) - Universidade Federal de Viçosa, Viçosa, MG, 2000.

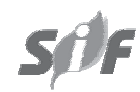

Revista Árvore, Viçosa-MG, v.38, n.4, p.725-732, 2014 
VALVERDE, S. R. et al. Efeitos

multiplicadores do setor florestal na

economia capixaba. Revista Árvore, v.29, n.1, p.85-93, 2005a.
VALVERDE, S. R. et al. Participação do Setor Florestal nos Indicadores Sócio-Econômicos do Estado do Espírito Santo. Revista Árvore, v.29, n.1, p.105-113, 2005b. 